\title{
Sparse channel estimation with regularization method using convolution inequality for entropy
}

\author{
Dongho Han, Sung-Phil Kim, Jose C. Principe, \\ Computational NeuroEngineering Lab. \\ Department of Electrical and Computer Engineering \\ University of Florida, Gainesville, FL 32611, USA \\ E-mail: \{dhan,phil,principe\}@ cnel.ufl.edu
}

\begin{abstract}
In this paper, we show that the sparse channel estimation problem can be formulated as a regularization problem between mean squared error(MSE) and the $L_{1}$-norm constraint of the channel impulse response. A simple adaptive method to solve regularization problem using the convolution inequality for entropy is proposed. Performance of this proposed regularization method will be compared to the Wiener filter, the matching pursuit (MP) algorithm and the information criterion based method. The results show that the estimate of the sparse channel using the MSE criterion with the $L_{1}$-norm constraint outperforms the Wiener filter and the conventional sparse solution methods in terms of MSE of the estimates and the generalization performance.
\end{abstract}

\section{INTRODUCTION}

Estimation of the sparse channel which has a small number of nonzero channel coefficients with a large span of delay is considered in this paper. Such sparse channels are encountered in many communication applications. High-Definition television(HDTV) channels are hundreds of data symbols long but there are only a few nonzero taps [1]. Hilly terrain delay profile has a small number of multipath in the broadband wireless communication [2] and underwater acoustic channels are also known to be sparse [3].

Estimation of the sparse channels proposed in [4] viewed this sparse channel estimation problem as a sparse representation problem and exploited the sparse nature of the channel using the matching pursuit (MP) algorithm and showed that it outperformed the least square based methods in robustness and low complexity accuracy. Matching pursuit algorithm is a method that has been used in many sparse applications [5],[6],[7]. Recently, a parametric method for selecting the structure of sparse model is proposed by Stoica [8]. This methods exploits the information provided by the local behavior of an information criterion. An adaptive method using the augmented Lagrangian to find the sparse solution has been proposed by Rao et. al [9]. This method augmented the $L_{1}$-norm constraint of the channel impulse response as a penalty term to the MSE criterion. It is well known that the concavity of the $L_{1}$-norm in the parameter space yields sparse solution and this sparsity measure has been widely used in many applications [6]. However, determination of the parameters in regularization problems, here $L_{1}$-norm of the channel coefficients which play an important role for good estimation, has been remained as an open problem.

In this paper, we propose a searching tool for the $L_{1}$-norm of channel coefficients using the convolution inequality for entropy [10], [11]. We will compare the performance of proposed sparse channel estimation method with the Wiener solution, the matching pursuit algorithm and the information criterion based method.

The organization of this paper is as follows: In the next section, we will summarize the proposed regularization method using the convolution inequality for entropy. In the following section, to help the readers understand the sparse problems, the conventional sparse methods such as the matching pursuit algorithm, and the information criterion based method are briefly introduced. Then, we will provide the experimental results of the comparison of channel estimation accuracy and generalization performance.

\section{SPARSE CHANNEL ESTIMATION}

A training sequence $u(n), n=0, \ldots, N-1$ is transmitted over the sparse channel, $h(0), h(1), \ldots, h(M-1)$ and white Gaussian noises $n(0), \ldots, n(N-1)$ are added to this transmitted signal. Then receive signal $z(n)$ can be expressed in matrix form as

$$
\mathbf{z}=\mathbf{A} \mathbf{h}+\mathbf{n}
$$

where

$$
\begin{gathered}
\mathbf{z}=\left(\begin{array}{c}
z(0) \\
\vdots \\
z(N-1)
\end{array}\right), \mathbf{h}=\left(\begin{array}{c}
h(0) \\
\vdots \\
h(M-1)
\end{array}\right), \mathbf{n}\left(\begin{array}{c}
n(0) \\
\vdots \\
n(N-1)
\end{array}\right) \\
\mathbf{A}=\left(\begin{array}{ccc}
u(0) & \ldots & u(-M+1) \\
\vdots & & \vdots \\
u(N-1) & \ldots & u(N-M)
\end{array}\right)
\end{gathered}
$$

The estimation of the sparse channel model, (1), can be stated as a approximation problem of the received signal vector $\mathbf{z}$ with a linear combination of a subset of columns in $\mathbf{A}$. 


\section{A. Sparse-LMS}

To find the sparse weight solution, Rao et. al [9] used $L_{1^{-}}$norm constraint of the weight vector. The cost function is

$$
J(\mathbf{h})=E\left(e_{k}^{2}\right)+\lambda\left[\sum_{i=1}^{L}\left|h_{i}\right|-\alpha\right]
$$

Where the first term is the mean squared error between the received signals and the estimated signals, and the second term is the penalty term. This constraint fixes the $L_{1}$-norm of the channel impulse response $\mathbf{h}$ to a constant $\alpha$. It is well known that the concavity of this $L_{1}$ norm function yields the sparse solution [6]. The penalty factor $\lambda$ can be included as an adaptive parameter by modifying the cost function as,

$$
J(\mathbf{h}, \lambda)=E\left(e_{k}^{2}\right)+\lambda \beta\left[\sum_{i=1}^{L}\left|h_{i}\right|-\alpha\right]-\lambda^{2} \beta
$$

where $\beta$ is a positive stabilization constant that keeps the penalty factor $\lambda$ bounded. We choose $\beta=1$ in the experiments. This modified cost function is known as the augmented Lagrangian [12]. The stochastic gradients of the cost function are given by,

$$
\begin{aligned}
\frac{\partial J(\mathbf{h}, \lambda)}{\partial h_{i}} & =-2 e_{k} u_{k i}+\lambda \beta\left[\operatorname{sign}\left(h_{i}\right)\right] \\
\frac{\partial J(\mathbf{h}, \lambda)}{\partial \lambda} & =\beta\left[\sum_{i=1}^{L}\left|h_{i}\right|-\alpha\right]-2 \beta \lambda
\end{aligned}
$$

Then the adaptation rule of parameters is given by

$$
\begin{aligned}
h_{i}(k+1) & =h_{i}(k)+\eta_{h}\left[2 e_{k} u_{k i}-\lambda_{k} \beta \operatorname{sign}\left(h_{i}\right)\right] \\
\lambda_{k+1} & =\lambda_{k}+\eta_{\lambda} \beta\left[\sum_{i=1}^{L}\left|h_{i}\right|-\alpha-2 \lambda_{k}\right]
\end{aligned}
$$

where $\eta_{h}$ and $\eta_{\lambda}$ are step size.

$\lambda_{k}$ converges to a value $\lambda^{*}$ [9]

$$
\lambda^{*}=\frac{1}{2}\left(\sum_{i=1}^{L}\left|h_{i}^{*}\right|-\alpha\right)
$$

where $h^{*}$ is the asymptotic coefficient vector from (6).

Note that the constant denoted by $\alpha$ in equation (2) is unknown so it has to be searched cautiously to get a good performance. With inappropriate presumption of $\alpha$, the performance of the adaptive system becomes poor. Therefore we propose a method based on the convolution inequality of entropy to determine $\alpha$ before learning system parameters using the stochastic gradient algorithm in equations (4) and (5).

\section{B. Convolution inequality for Renyi's entropy}

Consider a channel with $h(0), \ldots, h(M-1)$ and an independent identically distributed input $u(n)$. The output of the unknown linear system is $z(n)=\sum_{i} h_{i} u(n-i)$. Let
$H_{r}(\cdot)$ denote the Renyi's entropy then convolution inequality for Renyi's entropy is [10][11]

$$
H_{r}(Z) \geq H_{r}(U)+\log \left|h_{i}\right|, \forall i
$$

or equivalently,

$$
e^{H_{r}(Z)-H_{r}(U)} \geq \max \left|h_{i}\right|
$$

The equality holds if and only if the filter is a pure delay.

Let $h_{\max }$ denote the $\max \left|h_{i}\right|$, then we can start to search the adaptive parameter $\alpha$ in equation (2) using above equation (9). If the vector $\mathbf{h}$ is sparse, then $L_{1}$-norm of the channel impulse response will be not much bigger than $h_{\max }$. Instead of searching $\alpha$ in an arbitrary range, we can simplify searching based on this information. Therefore, we start searching with $\alpha=h_{\max }$, and train the adaptive filter with the algorithm given by equations (4) and (5). After training, we compute MSE between the received signals $\mathbf{z}$ and the filter outputs. Then, we increase $\alpha$ with some amount and repeat learning. After all iterations, we can find the value of $\alpha$ and the corresponding system parameters with the least MSE.

Since we cannot work directly with the PDF, nonparametric method is used here to estimate the entropy. Entropy estimate is obtained from Renyi's quadratic entropy estimator which estimates the PDF by Parzen-window method using Gaussian kernel [13], [14]. For instance, we calculate the entropy of output as:

$$
H_{R 2}(\hat{z})=-\log \left[\frac{1}{N^{2}} \sum_{i=1}^{N} \sum_{j=1}^{N} G\left(z_{i}-z_{j}, 2 \sigma^{2}\right)\right]
$$

where $\mathrm{N}$ is the number of sample, $G(\cdot)$ is the Gaussian kernel and $\sigma^{2}$ is its variance.

This proposed method can be summarized as follows:

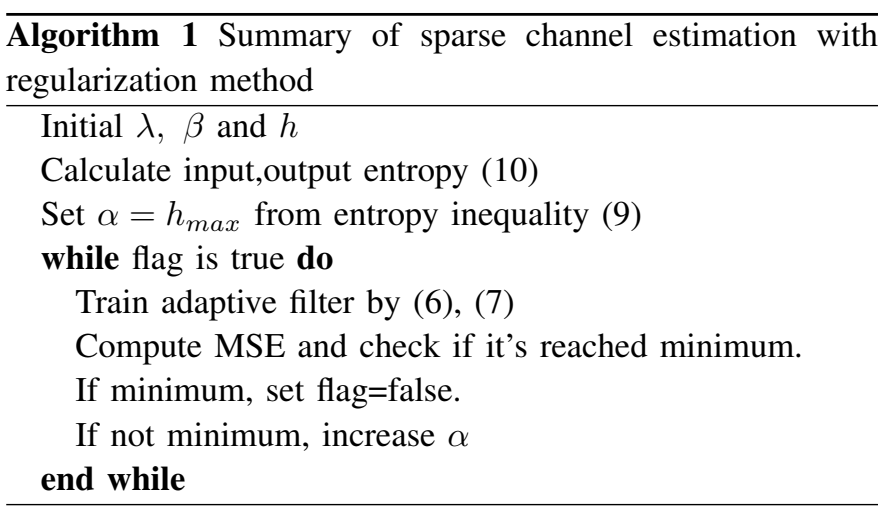

\section{OTHER SPARSE METHODS}

To help readers understand the sparse problem, we briefly summarize the conventional MP method [7] which have been widely used in many sparse application and recently proposed parametric method [8] which exploits the information provided by the local behavior of an information criterion. 


\section{A. Matching Pursuit based method}

The MP algorithm [7] first finds the best fitted column, $u_{k_{1}}$ in the matrix $\mathbf{A}$ with the received signal $z_{0}=z$. Then the projection of the initial residual $z_{0}$ along with the vector $u_{k_{1}}$ is removed from $z_{0}$ and the residual is $z_{1}$. Similarly, $u_{k_{2}}$ is decided that is best aligned to the residual $z_{1}$. This algorithm keeps finding the best aligned column to the consecutive residuals until a specified number of taps or small residuals. This algorithm is summarized as follows:

$$
\begin{array}{r}
k_{p}=\arg \max \left\|P_{u l} z_{p-1}\right\|=\arg \max \frac{\left|u_{l}^{H} z_{p-1}\right|^{2}}{\left\|u_{l}\right\|^{2}}, \\
l=1, \ldots, n, l \neq k_{p-1} \\
z_{p}=z_{p-1}-P_{u k_{p}} z_{p-1}=z_{p-1}-\frac{\left(u_{k_{p}}^{H} z_{p-1}\right) u_{k_{p}}}{\left\|u_{k_{p}}\right\|^{2}} \\
\hat{h}_{k_{p}}=\frac{\left(u_{k_{p}}^{H} z_{p-1}\right)}{\left\|u_{k_{p}}\right\|^{2}}
\end{array}
$$

where the projection onto vector $u_{l}$ is denoted as $P_{u_{l}}=\frac{u_{l} u_{l}^{H}}{\left\|u_{l}\right\|^{2}}$, the coefficient at position $k_{p}$ as $\hat{h}_{k_{p}}$.

\section{B. Information criterion based method}

If $G I C_{m_{k}}<G I C_{m_{k+1}}$, then the parameter vector of the $\mathrm{m}$ th order model $\hat{\theta}_{k+1}^{m}$ does not improve the model significantly from the model with $\hat{\theta}_{k}^{m}$ so we can set $\hat{\theta}_{k+1}^{m}=0$. By doing this we can select the sparse structure then we can adopt the LS solution to estimate the sparse channel by employing only the columns corresponds to nonzero coefficients. This is summarized as follows:

$$
\begin{aligned}
& G I C_{m_{k}}<G I C_{m_{k+1}} \rightarrow \hat{\theta}_{k+1}^{m}=0 \\
& G I C_{m_{k}}>G I C_{m_{k+1}} \rightarrow \hat{\theta}_{k+1}^{m} \neq 0
\end{aligned}
$$

where the generalized information criterion(GIC) is defined as:

$$
\begin{aligned}
G I C(m) & =-2 \ln p\left(\mathbf{z} \mid \hat{\theta}^{m}\right)+\eta \cdot m \\
\mathbf{z} & =\text { the available observed data } \\
\eta & =2 \text { for the Akaike information criterion } \\
& =\ln N \text { for the Bayesian information criterion }
\end{aligned}
$$

\section{Simulation RESUlts}

Nonzero coefficients of the sparse channels are drawn from a uniform distribution on $[-1,-0.2] \cup[0.2,1]$ and the number of the nonzero coefficients is 8 which is $10 \%$ of the channel length, $M=80$. These nonzero coefficients are positioned randomly over 80 taps. A white Gaussian training data with zero mean and unit variance is transmitted over the sparse channel and a white Gaussian noise with zero mean and variance of $\sigma_{n}^{2}$ is added to the received data. Variance $\sigma_{n}^{2}$ is varied to change the SNR from $5 \mathrm{~dB}$ to $30 \mathrm{~dB}$. The number of training data is $N=200.1000$ Monte Carlo simulations

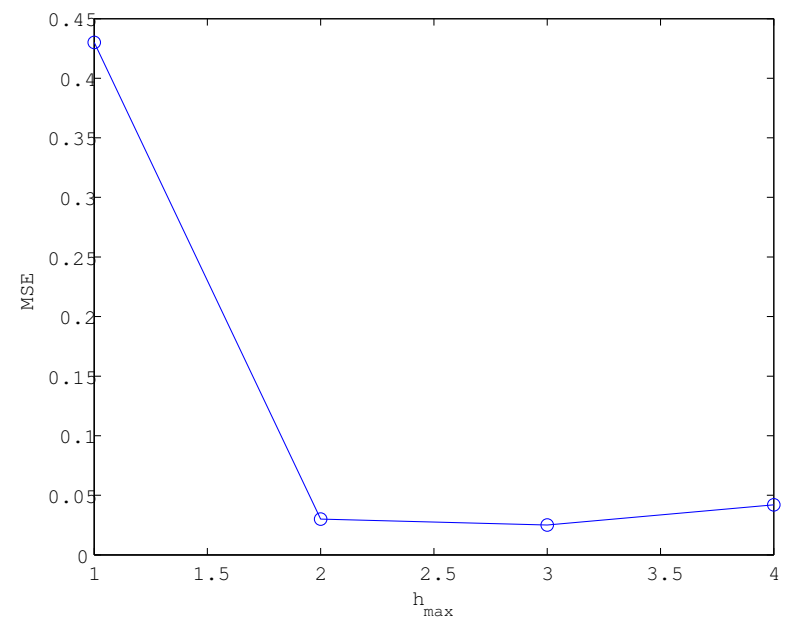

Fig. 1. Searching $\alpha$ using the proposed method based on convolution inequality for entropy.

with different input signals, SNR, and the nonzero channel coefficients are performed.

Table I shows the comparison of the proposed criterion performance to the Wiener's MSE criterion. We searched the $\alpha$ using the proposed method based on convolution inequality and fix the value of $\alpha$ to $\left(3 \cdot h_{\max }\right)$ from Fig. 1. Here, estimated $\alpha$ value is 5.5995 which is very close to the value of $L_{1}$ norm of true vector $\mathbf{h}, 5.6242$. As can be seen in Table I, the proposed method estimates the zero tap coefficients better than Wiener's MSE solution but there is a penalty for this. MSE is increased in nonzero tap coefficients estimation and it is the performance loss due to the penalty term.

We also compared the performance of the proposed method to matching pursuit algorithm which is widely used in sparse application and information criterion based method which is recently proposed. Fig. 2(a) shows that proposed method outperforms other methods in terms of the MSE of channel impulse response estimates. Fig. 2(b) shows that the generalization performance also outperforms other methods. To measure the generalization performance we feed the test data which is not used in training to the estimated channel. We can see from Fig. 2 that the performance of the proposed method at low SNR is much better than other methods except the MP method. Note that the iteration number of MP algorithm is 8 which is the exact number of nonzero channel tap in this simulation but number of nonzero taps are unknown in practical. So the stopping criterion of MP here (Fig. 2) is optimal.

\section{CONClusion}

We have employed the regularization method between MSE criterion and the $L_{1}$-norm constraint to identify the sparse channels with only a subset of coefficients are non-zero. In order to specify the range of search for the $L_{1}$-norm 


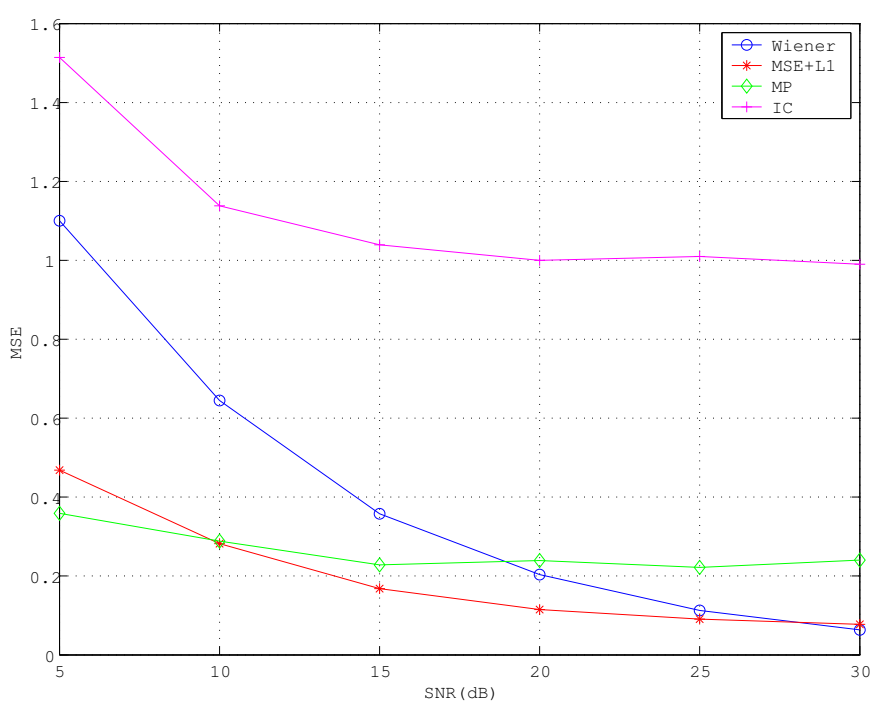

(a) MSE of the channel estimate

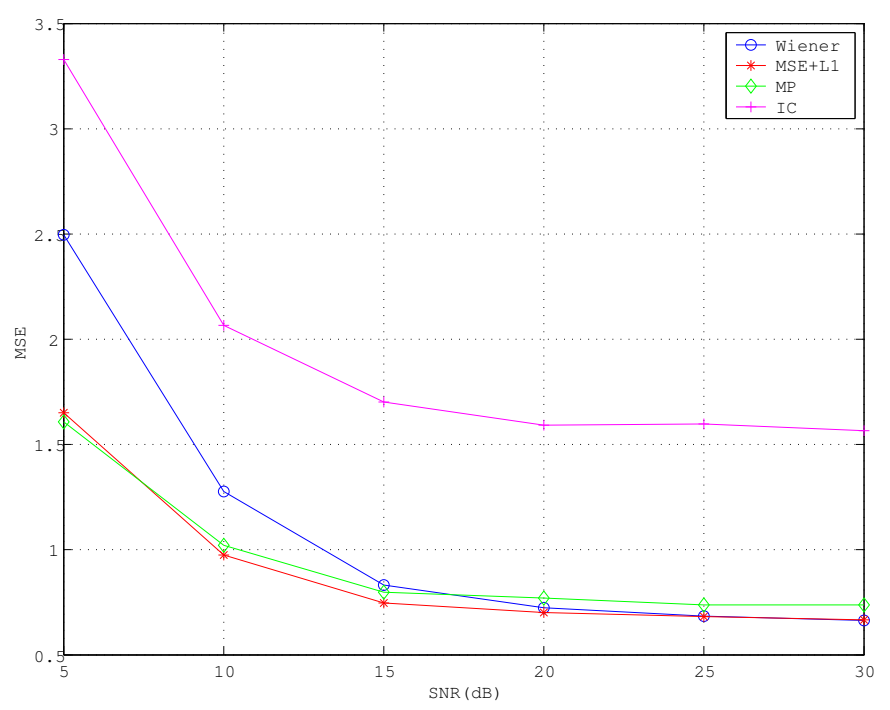

(b) generalization performance

Fig. 2. Performance comparison of proposed method with Wiener, MP and IC(Bayesian) based method

\begin{tabular}{|c|c|c|c|}
\hline & $\begin{array}{c}\text { overall tap } \\
\text { difference }\end{array}$ & $\begin{array}{c}\text { nonzero tap } \\
\text { difference }\end{array}$ & $\begin{array}{c}\text { zero tap } \\
\text { difference }\end{array}$ \\
\hline$\left|\mathbf{h}-\hat{\mathbf{h}}_{\text {wiener }}\right|$ & 0.6960 & 0.1746 & 0.6737 \\
$\left|\mathbf{h}-\hat{\mathbf{h}}_{\text {mse }+L_{1}}\right|$ & 0.2862 & 0.2425 & 0.1520 \\
\hline
\end{tabular}

TABLE I

DIFFERENCE BETWEEN THE TRUE WEIGHTS (h) AND ESTIMATED WEIGHTS $(\hat{\mathbf{h}})$ WITH THE PARAMETERS $\left(\mu_{h}=0.05, \mu_{\lambda}=0.01, \beta=1\right)$ WHEN $S N R=10 d B$.

of coefficient vector, we proposed to utilize the convolution inequality of Renyi's entropy. This adaptive system with regularization has demonstrated superior performance of the estimation of a sparse channel compared with Wiener filter and other sparse representation methods such as matching pursuit and the information criterion based method. We hope that this algorithm will provide practical tools to improve the sparse channel identification problem encountered in many real applications.

\section{ACKNOWLEDGMENT}

This work is partially supported by National Science Foundation grant ECS-0300340.

\section{REFERENCES}

[1] W. Schreiber, "Advanced television systems for terrestrial broadcasting: Some problems and some proposed solutions," in Proc. IEEE, vol. 83, June 1995, pp. 958-981.

[2] S. Ariyavisitakul, N. Sollenberger, and L. Greenstein, "Tap selectable decision-feedback equalization," IEEE Tran. on Commun., vol. 45, pp. 1497-1500, Dec 1997.

[3] M. Kocic et al., "Sparse equalization for real-time digital underwater acoustic communications," in Proc. OCEANS'95, San Diego,CA, Oct 1995, pp. 1417-1500.
[4] S. F. Cotter and B. D. Rao, "Sparse channel estimation via matching pursuit with application to equalization," IEEE Tran. on Communications, vol. 50, no. 3, pp. 374-377, 2002.

[5] B. D. Rao, "Signal processing with the sparseness constraint," in Proceedings of ICASSP'98, vol. III, Seattle, WA, May 1998, pp. 18611864.

[6] K. Kreutz-Delgado and B. D. Rao, "Sparse basis selection, ica, and majorization: towards a unified perspective," in Proceedings of ICASSP'99, vol. 2, March 1999, pp. 1081-1084.

[7] S.G.Mallat and Z.Zhang, "Matching pursuits with time-frequency dictionaries," IEEE Tran. on ASSP, vol. 41, no. 12, pp. 3397-3415, Dec 1993.

[8] P. Stoica, Y. Selen, and J. Li, "On information criteria and the generalized likelihood ratio test of model order selection," IEEE Signal Processing Letters, vol. 11, no. 10, Oct 2004.

[9] Y. N. Rao, S.-P. Kim, J. C. Sanchez, D. Erdogmus, J. C. Principe, J. M. Carmena, M. A. Lebedev, and M. A. Nicolelis, "Learning mappings in brain machine interfaces with echo state networks," to be appear in Proceedings of ICASSP'05.

[10] J.-F. Bercher and C. Vignat, "A renyi entropy convolution inequality with application," in Proceedings of EUSIPCO'02, vol. II, Tolouse, France, 2002, pp. 111-114.

[11] D. Erdogmus, K. E. Hild, J. C. Principe, M. Lazaro, and I. Santamaria, "Adaptive blind deconvolution of linear channels using renyi's entropy with parzen window estimation," IEEE Tran. on Signal Processing, vol. 52, no. 6, pp. 1489-1498, Jun 2004.

[12] D. Luenberger, Linear and Non-linear Programming. Addison Wesley, 1989.

[13] J. Principe, D. Xu, and J. Fisher, "Information theoretic learning," in Unsupervised Adaptive Filtering, S. Haykin, Ed. JOHN WILEY, 2000, pp. 265-319.

[14] J. Principe, N. Euliano, and W. Lefebvre, Neural and Adaptive Systems. JOHN WILEY, 2000 